\title{
Identification of genes and long non-coding RNAs associated with the pathogenesis of gastric cancer
}

\author{
ZHIWEI ZHAO ${ }^{1}$, YAN SONG $^{1}$, DAXUN PIAO ${ }^{2}$, TIANYOU LIU ${ }^{2}$ and LIANGLIANG ZHAO ${ }^{2}$ \\ ${ }^{1}$ Department of General Surgery, China-Japan Friendship Hospital of Jilin University, Changchun, Jilin 130033; \\ ${ }^{2}$ Department of General Surgery, The First Affiliated Hospital of Harbin Medical University, \\ Harbin, Heilongjiang 150001, P.R. China
}

Received March 28, 2015; Accepted June 5, 2015

DOI: $10.3892 /$ or.2015.4129

\begin{abstract}
Gastric cancer is a lethal disease characterized by high diffusivity and mortality. To examine the mechanisms involved in gastric cancer, we analyzed the microarray of GSE41476. GSE41476 was downloaded from the Gene Expression Omnibus and included 3 primary cell culture samples from gastric cancer tissues, 3 gastric cancer cell lines and 2 normal tissue samples. Long non-coding RNAs (lncRNAs) and differentially expressed genes (DEGs) were screened by Cuffdiff software. Functions of the DEGs were predicted by functional and pathway enrichment analyses. The interaction relationships of the proteins encoded by DEGs that were obtained from the STRING database and protein-protein interaction (PPI) network were visualized using Cytoscape. Modules analysis of PPI network was performed using CFinder. Moreover, IncRNA analysis was performed. A total of $86 \mathrm{lncRNAs}$, and 1,088 up- and 1,537 downregulated transcriptions were screened. For DEGs in module A of the PPI network for upregulated genes, the enriched pathways included ECM-receptor interaction and focal adhesion, both of which involved $C O L$ and $I T G$ genes. The $C O L$ genes interacted with the ITG genes (e.g., COL1A1-ITGA5 and COL1A2-ITGB1). For DEGs in module B of the PPI network for downregulated genes, the enriched pathways for DEGs
\end{abstract}

Correspondence to: Dr Yan Song, Department of General Surgery, China-Japan Friendship Hospital of Jilin University, 126 Xiantai Avenue, Changchun, Jilin 130033, P.R. China

E-mail: yansongysy@163.com

Abbreviations: ANXA1, Annexin A1; BP, biological process; CA IX, carbonic anhydrase IX; DEGs, differentially expressed genes; GKN2, gastrokine 2; GO, Gene Ontology; hg19, human genome 19; HOTAIR, HOX transcript antisense RNA; IL8, interleukin-8; lncRNAs, long non-coding RNAs; KEGG, Kyoto Encyclopedia of Genes and Genomes; MEG3, maternally expressed 3; PI3K, phosphatidylinositol 3-kinase; PPI, protein-protein interaction; PSCA, prostate stem cell antigen; suPAR, soluble urokinase plasminogen activator receptor

Key words: gastric cancer, differentially expressed genes, long non-coding RNAs, protein-protein interaction network, module analysis included the T-cell receptor signaling pathway, which involved PIK3CG and PIK3R5. PIK3CG had an interaction relationship with PIK3R5. In addition, IL7 was co-expressed with TCONS00068220. In summary, the results showed that $C O L$ and $I T G$ genes, PIK3CG, PIK3R5, IL7 and IncRNA TCONS-00068220 may play a role in gastric cancer.

\section{Introduction}

As a type of malignant epithelial tumor, gastric cancer is derived from the glandular epithelium of the gastric mucosa (1). Gastric cancer has high diffusivity, such as spread to lungs, liver and bones (2). This cancer type is prevalent in men of developing countries $(3,4)$, and $\sim 8.5 \%$ of cancer cases in men is gastric cancer (5). In 2012, gastric cancer ranked third after lung and liver cancer, with 700,000 mortalities $(6,7)$. Thus, it is necessary to examine the molecular mechanisms of gastric cancer and develop therapeutic schedules.

The molecular mechanisms of gastric cancer have been previously investigated. For example, as a member of the BRICHO family, the full-length gene gastrokine 2 (GKN2) is downregulated and associated with gastric cancer (8). The expression of GKN1 and GKN2 decreased in gastric adenocarcinomas, and their loss is associated with shorter survival in the intestinal subtype of gastric adenocarcinomas (9). Expression of soluble urokinase plasminogen activator receptor $(s u P A R)$ and carbonic anhydrase IX $(C A I X)$ is associated with the stage and presence of gastric cancer, and the overexpression of $s U P A R$ indicates a poorer prognosis in patients with gastric cancer (10). Differentially expressed microseminoprotein $(M S M B)$, Annexin A10 (ANXA10), Annexin A1 (ANXAl) and prostate stem cell antigen (PSCA) have been identified in normal and cancer gastric tissues, and may be used as biomarkers for the diagnosis and treatment of gastric cancer (11).

Long non-coding RNAs (lncRNAs), having a length $>200$ nucleotides, can regulate gene expression during transport, RNA maturation and protein synthesis (12). Dysregulation of lncRNAs is associated with many types of human cancer (13). Many differentially expressed lncRNAs, such as LINC00152 and LINC00261, have been identified and may act as therapeutic targets and biomarkers in gastric cancer (12). The downregulation of IncRNA maternally 
Table I. The top 10 enriched GO functions and KEGG pathways for the DEGs.

\begin{tabular}{|c|c|c|c|c|c|}
\hline Genes & ID & Name & Gene no. & Gene symbol & P-value \\
\hline \multicolumn{6}{|l|}{$\mathrm{GO}$ functions } \\
\hline \multirow[t]{5}{*}{ Upregulated } & GO:0030198 & $\begin{array}{l}\text { Extracellular matrix } \\
\text { organization }\end{array}$ & 71 & ACTN1, ADAM17... & 0 \\
\hline & GO:0043062 & $\begin{array}{l}\text { Extracellular structure } \\
\text { organization }\end{array}$ & 71 & ADAM9, ADAMTS2... & 0 \\
\hline & GO:0016477 & Cell migration & 110 & AJUBA, AMOTL1 ... & $8.97 \mathrm{E}-14$ \\
\hline & GO:0031012 & Extracellular matrix & 422 & ADAMTS1, CD248... & $3.06 \mathrm{E}-14$ \\
\hline & GO:0005515 & Protein binding & 521 & $A B L 2, A B L I M 3 \ldots$ & 3.37E-11 \\
\hline \multirow[t]{5}{*}{ Downregulated } & GO:0048584 & $\begin{array}{l}\text { Positive regulation of response } \\
\text { to stimulus }\end{array}$ & 189 & $A 2 M, A D A \ldots$ & $6.66 \mathrm{E}-16$ \\
\hline & GO:0044699 & Single-organism process & 1017 & $E P H X 2, E P N 1 \ldots$ & $1.33 \mathrm{E}-15$ \\
\hline & GO:0002694 & $\begin{array}{l}\text { Regulation of leukocyte } \\
\text { activation }\end{array}$ & 76 & $A I F 1, B C R \ldots$ & $1.89 \mathrm{E}-15$ \\
\hline & GO:0031224 & Intrinsic to membrane & 565 & $A 4 G N T, A A D A C \ldots$ & $1.11 \mathrm{E}-15$ \\
\hline & GO:0005102 & Receptor binding & 146 & $A P L P 1, A P O E \ldots$ & $4.68 \mathrm{E}-08$ \\
\hline \multicolumn{6}{|l|}{ KEGG pathways } \\
\hline \multirow[t]{5}{*}{ Upregulated } & KEGG:4512 & ECM-receptor interaction & 20 & CD44, COL1A1... & $1.18 \mathrm{E}-06$ \\
\hline & KEGG:4510 & Focal adhesion & 29 & ACTN1, COL1A1 ... & 0.000143 \\
\hline & KEGG:5146 & Amoebiasis & 18 & RKCA, SHC3... & 0.000392 \\
\hline & KEGG:5144 & Malaria & 11 & $C C L 2, C S F 3 \ldots$ & 0.000695 \\
\hline & KEGG:1040 & $\begin{array}{l}\text { Biosynthesis of unsaturated } \\
\text { fatty acids }\end{array}$ & 6 & $P T P L A, S C D \ldots$ & 0.00262 \\
\hline \multirow[t]{5}{*}{ Downregulated } & KEGG:4514 & Cell adhesion molecules (CAMs) & 46 & $C D 2, C D 22 \ldots$ & $1.33 \mathrm{E}-15$ \\
\hline & KEGG:5150 & Staphylococcus aureus infection & 23 & $D Q A 1, H L A-D Q A 2 \ldots$ & $2.53 \mathrm{E}-10$ \\
\hline & KEGG:4672 & $\begin{array}{l}\text { Intestinal immune network } \\
\text { for IgA production }\end{array}$ & 19 & ICAM $2, I C A M 3 \ldots$ & $2.80 \mathrm{E}-08$ \\
\hline & KEGG:5340 & Primary immunodeficiency & 15 & $A D A, B L N K \ldots$ & $2.40 \mathrm{E}-07$ \\
\hline & KEGG:5416 & Viral myocarditis & 22 & $B I D, C D 28 \ldots$ & $2.86 \mathrm{E}-07$ \\
\hline
\end{tabular}

GO, Gene Ontology; KEGG, Kyoto Encyclopedia of Genes and Genomes; DEGs, differentially expressed genes.

expressed $3(M E G 3)$ is involved with cell proliferation and can be regarded as a poor prognostic biomarker in gastric cancer (14). Knockdown of lncRNA HOX transcript antisense RNA (HOTAIR) results in suppression of tumor invasion and reversal of epithelial-mesenchymal transition process in gastric cancer cells, suggesting that HOTAIR affects diagnostics and therapeutics of gastric cancer (15).

In the present study, to examine the molecular mechanisms of gastric cancer, the expression profile of GSE41476 was downloaded, which involved 3 primary cell culture samples from gastric cancer tissues, 3 gastric cancer cell lines and 2 normal tissue samples. The lncRNAs were predicted and differentially expressed genes (DEGs) were screened. The functions of the DEGs were analysed using Gene Ontology (GO) and pathway enrichment analyses. In addition, a search was conducted to determine the interaction relationships between the DEGs using protein-protein interaction (PPI) network and modules of the PPI network. Additionally, lncRNA-DEG pairs were screened, and pathway enrichment analysis was performed for DEGs co-expressed with each IncRNA.

\section{Materials and methods}

Microarray data. The expression profile of GSE41476 was downloaded from the Gene Expression Omnibus (GEO, http:/ www.ncbi.nlm.nih.gov/geo/), which was based on the platform of the GPL9115 Illumina Genome Analyzer II (Homo sapiens). GSE41476 included a collective of 3 primary cell culture samples from gastric cancer tissues, 3 gastric cancer cell lines and 2 normal tissue samples.

Sequence alignment. After GSE41476 was downloaded, the SRA format sequences were translated into FASTQ format, and microarray data were preprocessed by NGSQC software (16). The ratio of bases with base sequencing quality $<20$ was required to be $<0.1$. The remaining high quality sequences were compared to human genome 19 (hg19) using TopHat2 (17). The parameter was set to - no-discordant - phred64-quals, and the remaining parameters were set to the default values.

IncRNA prediction. The alignment results were obtained via transcriptome assembly using Cufflinks software (18). 
Subsequently, the assembly results were integrated using Cuffmerge software (19). According to the gene annotation information of the genome in the UCSC, assembly results that did not overlap with the extracted arbitrary genes were extracted. Transcriptions with a length of $>200 \mathrm{nt}$ and with $\geq 2$ exons were screened. According to information obtained from the 29 mammalian genome alignment, transcriptions with scores $<100$ were screened using PhyloCSF software (20). Moreover, HMMER software was used to compare transcriptions to Pfam database (21). E-value $<1 \mathrm{e}-5$ was used as the cut-off criterion.

DEGs screening. A search through the RefSeq annotation files in UCSC website identified known lncRNA comments (LNCipedia1.0 database) and the predicted lncRNA transcription document, DEGs and lncRNAs, which were screened from the alignment results using Cuffdiff software (22). The adjusted p-value of $<0.05$ and $\mid \log$ fold-change (FC) $\mid>1$ were used as the cut-off criteria.

Functional and pathway enrichment analyses. As a functional study method, GO analysis is used to assess large-scale transcriptomic or genomic data (23). The Kyoto Encyclopedia of Genes and Genomes (KEGG) pathway database shows how molecules or genes act (24). The GO and KEGG pathway enrichment analyses were conducted for DEGs. The GO functional enrichment analysis was mainly focused on the biological process (BP). $\mathrm{P}<0.05$ was used and $\geq 2$ genes were used as the cut-off criteria.

PPI network and module analysis. The STRING online software (25) was used to examine the interaction relationships of the proteins encoded by DEGs, and the combined score $>0.7$ was used as the cut-off criterion. The Cytoscape software (26) was used to visualize the PPI network. The CFinder software (27) was used to screen modules of the PPI network, and the parameter $\mathrm{k}$ was set to 8 .

lncRNA analysis. According to the expression matrix of the differentially expressed lncRNA and the DEGs, the relationship between lncRNA and the DEGs was calculated and IncRNA-DEG pairs were screened. Pearson's correlation of $>0.99$ was used as the cut-off criterion.

\section{Results}

lncRNA prediction and DEGs analysis. A total of 86 lncRNA transcriptions were obtained, including 37 transcriptions with an overlap of $>50 \%$ when compared with known lncRNAs.

Compared to normal tissue samples, 1,088 upregulated transcriptions (including 16 known lncRNAs, 1 predicted lncRNAs and 1,071 mRNAs) and 1,537 downregulated transcriptions (including 18 known lncRNAs, 4 predicted lncRNAs and 1,515 mRNAs) were identified in the gastric cancer samples.

Functional and pathway enrichment analyses. The enriched GO functions for the DEGs are provided in Table I, including cell migration $(\mathrm{P}=8.97 \mathrm{E}-14)$, extracellular matrix $(\mathrm{P}=3.06 \mathrm{E}-14)$, positive regulation of response to stimulus $(\mathrm{P}=6.66 \mathrm{E}-16)$ and single-organism process $(\mathrm{P}=1.33 \mathrm{E}-15)$.
The enriched KEGG pathways for the DEGs are shown in Table I, including ECM-receptor interaction ( $\mathrm{P}=1.18 \mathrm{E}-06)$, focal adhesion $(\mathrm{P}=0.000143)$, cell adhesion molecules (CAMs, $\mathrm{P}=1.33 \mathrm{E}-15)$ and Staphylococcus aureus infection $(\mathrm{P}=2.53 \mathrm{E}-10)$.

PPI network and module analysis. The PPI network of the upregulated genes had 568 nodes and 1,522 interactions (Fig. 1). Module A (Fig. 2A) and module B (Fig. 2B) were obtained from the PPI network. Module A had 13 nodes and 65 interactions. The enriched GO functions for DEGs in module A are provided in Table II, including collagen catabolic process $(\mathrm{P}=2.22 \mathrm{E}-16)$, multicellular organismal catabolic process $(\mathrm{P}=6.66 \mathrm{E}-16)$ and extracellular matrix disassembly $(\mathrm{P}=1.33 \mathrm{E}-$ 15). The enriched KEGG pathways for DEGs in module A are also listed in Table II, including ECM-receptor interaction $[\mathrm{P}=0$, which involved proteins encoded by collagen $(C O L)$ genes such as collagen, type I, $\alpha 1(C O L 1 A 1)]$, collagen, type I, $\alpha 2$ (COL1A2) and collagen, type IV, $\alpha 4$ (COL4A4), as well as proteins encoded by integrin $(I T G)$ genes such as integrin, $\alpha 1$ (ITGA1), integrin, $\alpha 5$ (ITGA5) and integrin, $\beta 1$ (ITGB1), focal adhesion $(\mathrm{P}=1.05 \mathrm{E}-13$, which also involved proteins encoded by the $C O L$ and $I T G$ genes) and protein digestion and absorption $(\mathrm{P}=5.49 \mathrm{E}-11)$. Proteins encoded by $C O L$ genes interacted with those of the ITG genes, such as COL1A1-ITGA5 and COL1A2-ITGB1. Module B had 27 nodes and 261 interactions. The enriched GO functions for DEGs in module B are provided in Table II, including ribonucleoprotein complex biogenesis $(\mathrm{P}=2.22 \mathrm{E}-16)$, rRNA processing $(\mathrm{P}=1.17 \mathrm{E}-11)$ and cellular component biogenesis $(\mathrm{P}=2.36 \mathrm{E}-07)$. The enriched KEGG pathway for DEGs in module $\mathrm{B}$ was ribosome biogenesis in eukaryotes $(\mathrm{P}=5.55 \mathrm{E}-16)$ (Table II).

The PPI network of the downregulated genes had 734 nodes and 2345 interactions (Fig. 3). In addition, 6 modules (module A-F) were obtained from the PPI network (Fig. 4). Module A had 8 nodes and 28 interactions. The enriched GO functions for DEGs in module A included transcription initiation from RNA polymerase II promoter $(\mathrm{P}=8.88 \mathrm{E}-16)$ and DNA-dependent transcription, initiation $(\mathrm{P}=2.66 \mathrm{E}-15)$. The enriched KEGG pathways for DEGs in module $\mathrm{A}$ included maturity onset diabetes of the young $(\mathrm{P}=0.000173)$ and bile secretion $(\mathrm{P}=0.001409)$. Module $\mathrm{B}$ had 20 nodes and 122 interactions. The enriched GO functions for DEGs in module $\mathrm{B}$ included activation of immune response $(\mathrm{P}=2.22 \mathrm{E}-16)$ and leukocyte activation $(\mathrm{P}=4.44 \mathrm{E}-16)$. The enriched KEGG pathways for DEGs in module $\mathrm{B}$ included T-cell receptor signaling pathway $[\mathrm{P}=0$, which involved phosphoinositide-3-kinase, catalytic, $\gamma$ polypeptide (PIK3CG) and phosphoinositide-3-kinase, regulatory subunit 5 (PIK3R5)] and primary immunodeficiency $(\mathrm{P}=7.35 \mathrm{E}-10)$. Specifically, PIK3CG had an interaction relationship with PIK3R5. Module $\mathrm{C}$ had 8 nodes and 28 interactions. The enriched GO functions for DEGs in module $\mathrm{C}$ included cytokinemediated signaling pathway $(\mathrm{P}=6.98 \mathrm{E}-14)$ and cell response to cytokine stimulus $(\mathrm{P}=5.20 \mathrm{E}-13)$. The enriched KEGG pathways for $\mathrm{DEG}$ in module $\mathrm{C}$ included antigen processing and presentation $(\mathrm{P}=0.001613)$ and hepatitis $\mathrm{C}(\mathrm{P}=0.004944)$. Module D had 10 nodes and 44 interactions. The enriched GO functions for DEGs in module D included single-organism carbohydrate metabolic process $(\mathrm{P}=8.44 \mathrm{E}-15)$ and keratan 


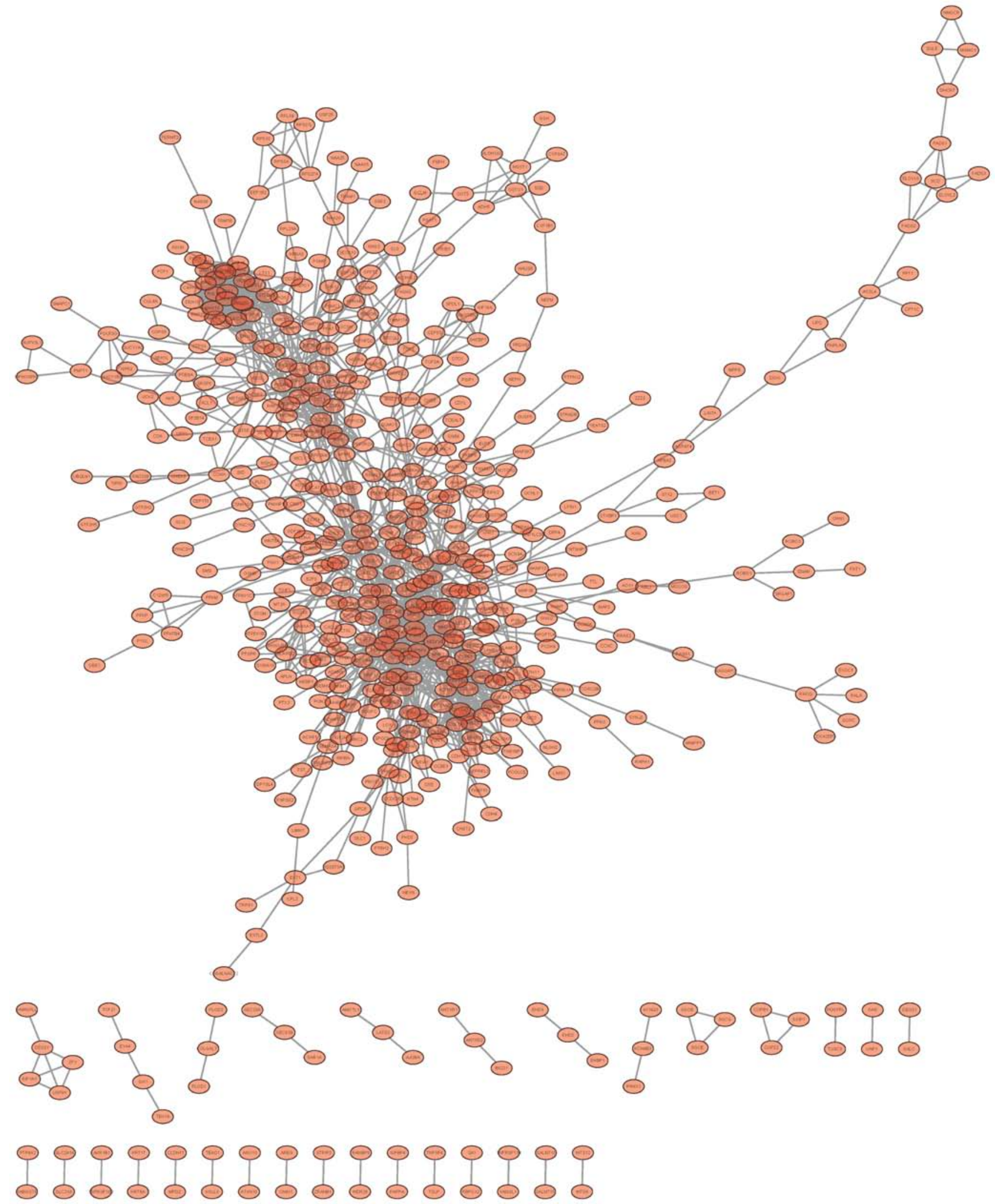

Figure 1. Protein-protein interaction network of the upregulated genes.

sulfate biosynthetic process $(\mathrm{P}=0.000153)$. The enriched KEGG pathways for DEGs in module D included mucin-type O-glycan biosynthesis $(\mathrm{P}=2.39 \mathrm{E}-06)$ and metabolic pathways $(\mathrm{P}=0.014715)$. Module $\mathrm{E}$ had 8 nodes and 28 interactions. The enriched GO functions for DEGs in module E included T-cell costimulation ( $\mathrm{P}=1.20 \mathrm{E}-10)$. The enriched KEGG pathways for DEGs in module $\mathrm{E}$ included $\mathrm{T}$-cell receptor signaling pathway ( $\mathrm{P}=3.92 \mathrm{E}-08)$. Module $\mathrm{F}$ had 24 nodes and 273 interactions. The enriched GO functions for DEGs in module $\mathrm{F}$ included signal transduction $(\mathrm{P}=1.46 \mathrm{E}-13)$. The enriched 
Table II. The top 10 enriched GO functions and KEGG pathways for DEGs in module A and B of the PPI network for the upregulated DEGs.

\begin{tabular}{|c|c|c|c|c|c|}
\hline Modules & ID & Name & $\begin{array}{c}\text { Gene } \\
\text { no. }\end{array}$ & $\begin{array}{l}\text { Gene } \\
\text { symbol }\end{array}$ & P-value \\
\hline \multicolumn{6}{|c|}{ GO functions } \\
\hline \multirow[t]{5}{*}{ A } & GO:0030574 & Collagen catabolic process & 8 & COL5A2, COL $4 A 4 \ldots$ & $2.22 \mathrm{E}-16$ \\
\hline & GO:0044243 & $\begin{array}{l}\text { Multicellular organismal catabolic } \\
\text { process }\end{array}$ & 8 & COL $4 A 4, C O L 1 A 2 \ldots$ & $6.66 \mathrm{E}-16$ \\
\hline & GO:0022617 & Extracellular matrix disassembly & 8 & $C O L 1 A 2, C O L 1 A 1 \ldots$ & $1.33 \mathrm{E}-15$ \\
\hline & GO:0005581 & Collagen & 8 & COL5A2, COL $4 A 4$ & 4.44E-16 \\
\hline & GO:0005201 & $\begin{array}{l}\text { Extracellular matrix structural } \\
\text { constituent }\end{array}$ & 5 & $C O L 1 A 1, C O L 12 A 1 \ldots$ & 4.23E-09 \\
\hline \multirow[t]{5}{*}{$\mathrm{B}$} & GO:0022613 & $\begin{array}{l}\text { Ribonucleoprotein complex } \\
\text { biogenesis }\end{array}$ & 11 & BRIX1, TSR $1 \ldots$ & $2.22 \mathrm{E}-16$ \\
\hline & GO:0006364 & rRNA processing & 7 & WDR12, NOP58... & $1.17 \mathrm{E}-11$ \\
\hline & GO:0044085 & Cellular component biogenesis & 11 & $B R I X 1, T S R 1 \ldots$ & $2.36 \mathrm{E}-07$ \\
\hline & GO:0031981 & Nuclear lumen & 21 & $B R I X 1, T S R 1 \ldots$ & $1.33 \mathrm{E}-15$ \\
\hline & GO:0003723 & RNA binding & 7 & $P N O 1, D K C 1 \ldots$ & 4.48E-06 \\
\hline \multicolumn{6}{|c|}{ KEGG pathways } \\
\hline \multirow[t]{9}{*}{ A } & KEGG:4512 & ECM-receptor interaction & 11 & $\begin{array}{l}\text { COL } 5 A 2, \text { COL } 4 A 4 \\
\text { COL } 1 A 2 \ldots\end{array}$ & 0 \\
\hline & KEGG:4510 & Focal adhesion & 10 & $\begin{array}{l}\text { COL } 5 A 2, \text { COL } 4 A 4, \\
\text { COL } 1 A 2 \ldots\end{array}$ & $1.05 \mathrm{E}-13$ \\
\hline & KEGG:4974 & Protein digestion and absorption & 7 & $\begin{array}{l}\text { COL } 5 A 2, \text { COL } 4 A 4 \\
\text { COL } 1 A 2 \ldots\end{array}$ & $5.49 \mathrm{E}-11$ \\
\hline & KEGG:5412 & $\begin{array}{l}\text { Arrhythmogenic right ventricular } \\
\text { cardiomyopathy (ARVC) }\end{array}$ & 4 & $\begin{array}{l}\text { ITGB1, ITGA1, } \\
\text { ITGA5, ITGB5 }\end{array}$ & $1.07 \mathrm{E}-05$ \\
\hline & KEGG:5410 & $\begin{array}{l}\text { Hypertrophic cardiomyopathy } \\
\text { (HCM) }\end{array}$ & 4 & $\begin{array}{l}\text { ITGB } 1, \text { ITGA1, } \\
\text { ITGA5, ITGB5 }\end{array}$ & $1.69 \mathrm{E}-05$ \\
\hline & KEGG:5414 & Dilated cardiomyopathy & 4 & $\begin{array}{l}I T G B 1, I T G A 1 \\
I T G A 5, I T G B 5\end{array}$ & 2.33E-05 \\
\hline & KEGG:5146 & Amoebiasis & 4 & $\begin{array}{l}\text { COL 5A2, COL } 4 A 4 \\
\text { COL1A2, COL1A1 }\end{array}$ & 4.45E-05 \\
\hline & KEGG:5131 & Shigellosis & 3 & $\begin{array}{l}\text { ITGB1, ITGA5, } \\
C D 44\end{array}$ & 0.000219795 \\
\hline & KEGG:4640 & Hematopoietic cell lineage & 3 & $\begin{array}{l}\text { ITGB1, ITGA5, } \\
C D 44\end{array}$ & 0.000649577 \\
\hline $\mathrm{B}$ & KEGG:3008 & Ribosome biogenesis in eukaryotes & 9 & $\begin{array}{l}\text { NOP58, GTPBP } 4, \text { WDR3, } \\
\text { WDR75, UTP15, WDR36, } \\
\text { DKC } 1, \text { CIRH1A, WDR43 }\end{array}$ & $5.55 \mathrm{E}-16$ \\
\hline
\end{tabular}

GO, Gene Ontology; KEGG, Kyoto Encyclopedia of Genes and Genomes; DEGs, differentially expressed genes; PPI, protein-protein interaction.
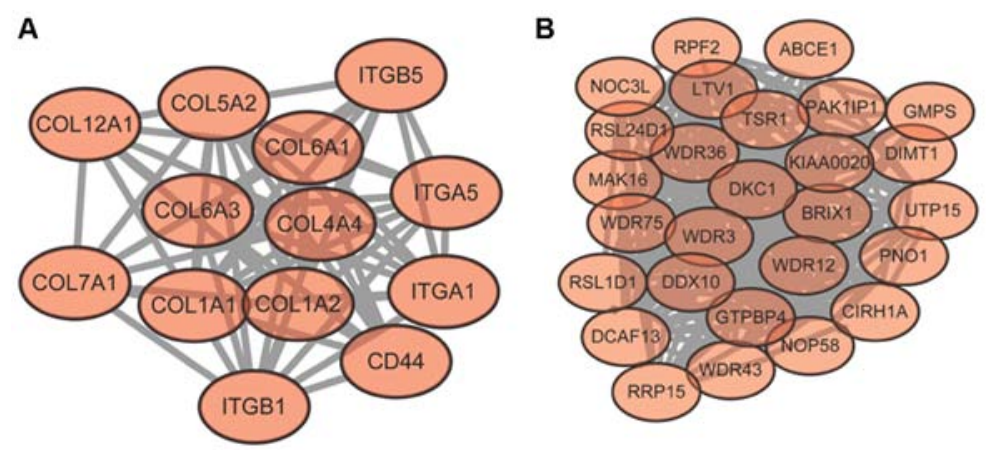

Figure 2. Module A and B obtained from the PPI network of the upregulated genes. PPI, protein-protein interaction. 
Table III. The top 10 enriched GO functions and KEGG pathways for DEGs in module A, B, C, D, E and F of the PPI network for the downregulated DEGs.

\begin{tabular}{|c|c|c|c|c|c|}
\hline Modules & ID & Name & $\begin{array}{c}\text { Gene } \\
\text { no. }\end{array}$ & $\begin{array}{l}\text { Gene } \\
\text { symbol }\end{array}$ & P-value \\
\hline \multicolumn{6}{|c|}{ GO functions } \\
\hline \multirow[t]{2}{*}{ A } & GO:0006367 & $\begin{array}{l}\text { Transcription initiation from RNA } \\
\text { polymerase II promoter }\end{array}$ & 8 & $E S R R G, E S R R B \ldots$ & $8.88 \mathrm{E}-16$ \\
\hline & GO:0006352 & $\begin{array}{l}\text { DNA-dependent transcription, } \\
\text { initiation }\end{array}$ & 8 & $N R O B 2, H N F 4 G \ldots$ & $2.66 \mathrm{E}-15$ \\
\hline \multirow[t]{2}{*}{$\mathrm{B}$} & GO:0002253 & Activation of immune response & 13 & $C D 3 D, C D 3 E \ldots$ & $2.22 \mathrm{E}-16$ \\
\hline & GO:0045321 & Leukocyte activation & 14 & $C D 3 D, C D 3 E \ldots$ & 4.44E-16 \\
\hline \multirow[t]{2}{*}{$\mathrm{C}$} & GO:0019221 & $\begin{array}{l}\text { Cytokine-mediated signaling } \\
\text { pathway }\end{array}$ & 8 & IRF7, CIITA ... & $6.98 \mathrm{E}-14$ \\
\hline & GO:0071345 & Cellular response to cytokine stimulus & 8 & IRF7, CIITA ... & $5.20 \mathrm{E}-13$ \\
\hline \multirow[t]{2}{*}{$\mathrm{D}$} & GO:0044723 & $\begin{array}{l}\text { Single-organism carbohydrate } \\
\text { metabolic process }\end{array}$ & 10 & 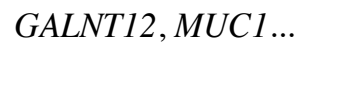 & $8.44 \mathrm{E}-15$ \\
\hline & GO:0018146 & Keratan sulfate biosynthetic process & 2 & B3GNT7, B3GNT3 & 0.000153 \\
\hline $\mathrm{E}$ & GO:0031295 & T-cell costimulation & 5 & $\begin{array}{l}C D 4, C D 247, C D 3 D \\
C D 3 E, C D 3 G\end{array}$ & $1.20 \mathrm{E}-10$ \\
\hline $\mathrm{F}$ & GO:0007165 & Signal transduction & 24 & CCL5, ADORA3... & $1.46 \mathrm{E}-13$ \\
\hline \multicolumn{6}{|c|}{ KEGG pathways } \\
\hline \multirow[t]{2}{*}{ A } & KEGG: 04950 & Maturity onset diabetes of the young & 2 & $H N F 4 G, H N F 4 A$ & 0.000173 \\
\hline & KEGG:04976 & Bile secretion & 2 & $N R O B 2, R X R A$ & 0.001409 \\
\hline \multirow[t]{2}{*}{$\mathrm{B}$} & KEGG:4660 & T-cell receptor signaling pathway & 13 & $C D 3 D, C D 3 E, C D 3 G \ldots$ & 0 \\
\hline & KEGG:5340 & Primary immunodeficiency & 6 & $C D 3 D, C D 3 E, C D 4 \ldots$ & $7.35 \mathrm{E}-10$ \\
\hline \multirow[t]{2}{*}{$\mathrm{C}$} & KEGG:4612 & Antigen processing and presentation & 2 & CIITA, IFI30 & 0.001613 \\
\hline & KEGG:5160 & Hepatitis C & 2 & IRF7, OAS3 & 0.004944 \\
\hline \multirow[t]{2}{*}{$\mathrm{D}$} & KEGG:512 & Mucin-type O-glycan biosynthesis & 3 & $\begin{array}{l}\text { COL5A2, COLAA4, } \\
\text { COL1A2, COL1A1 }\end{array}$ & 2.39E-06 \\
\hline & KEGG:1100 & Metabolic pathways & 4 & ITGB1, ITGA5, CD44 & 0.014715 \\
\hline $\mathrm{E}$ & KEGG:4660 & T-cell receptor signaling pathway & 5 & $\begin{array}{l}C D 4, C D 247, C D 3 D \\
C D 3 E, C D 3 G\end{array}$ & $3.92 \mathrm{E}-08$ \\
\hline $\mathrm{F}$ & KEGG:4080 & Neuroactive ligand-receptor interaction & 9 & $\begin{array}{l}\text { ADORA3, C5AR1, } \\
\text { C3AR1... }\end{array}$ & $9.26 \mathrm{E}-08$ \\
\hline
\end{tabular}

GO, Gene Ontology; KEGG, Kyoto Encyclopedia of Genes and Genomes; DEGs, differentially expressed genes; PPI, protein-protein interaction.

KEGG pathways for DEGs in module $\mathrm{F}$ included neuroactive ligand-receptor interaction $(\mathrm{P}=9.26 \mathrm{E}-08)$ (Table III).

lncRNA analysis. After IncRNA-DEG pairs, such as TCONS00068220-IL7, were screened, KEGG pathway enrichment analysis was conducted for DEGs co-expressed with each IncRNA. Proteins encoded with co-expressed DEGs of TCONS_00068220 were enriched in cancer-related pathways, such as bladder cancer, CAMs, chemokine signaling pathway and natural killer cell-mediated cytotoxicity (Table IV) (28).

\section{Discussion}

In the present study, 86 lncRNA transcriptions were obtained, including 37 transcriptions with an overlap of $>50 \%$ when compared with known lncRNAs. Additionally, 1,088 upregulated and 1,537 downregulated transcriptions were screened. The functions of cell migration, positive regulation of response to stimulus and single-organism process were enriched for the DEGs.

At the tumor periphery of scirrhous gastric carcinoma, collagen biosynthesis is increased and may contribute to the invasion of tumor cells (29). Collagen IV can play a role in cell adhesion, as well as tumor metastasis and invasion (30-32). It is reported that integrin (ITG) $\alpha 2 \beta 1$ can be used as a candidate target molecule involved in the prevention of gastric cancer peritoneal dissemination (33). Integrin $\alpha 6 \beta 4$ is a suppressor and can be a biomarker for peritoneal dissemination in gastric cancer (34). In module A of the PPI network for the upregulated genes, the enriched KEGG pathways for DEGs 


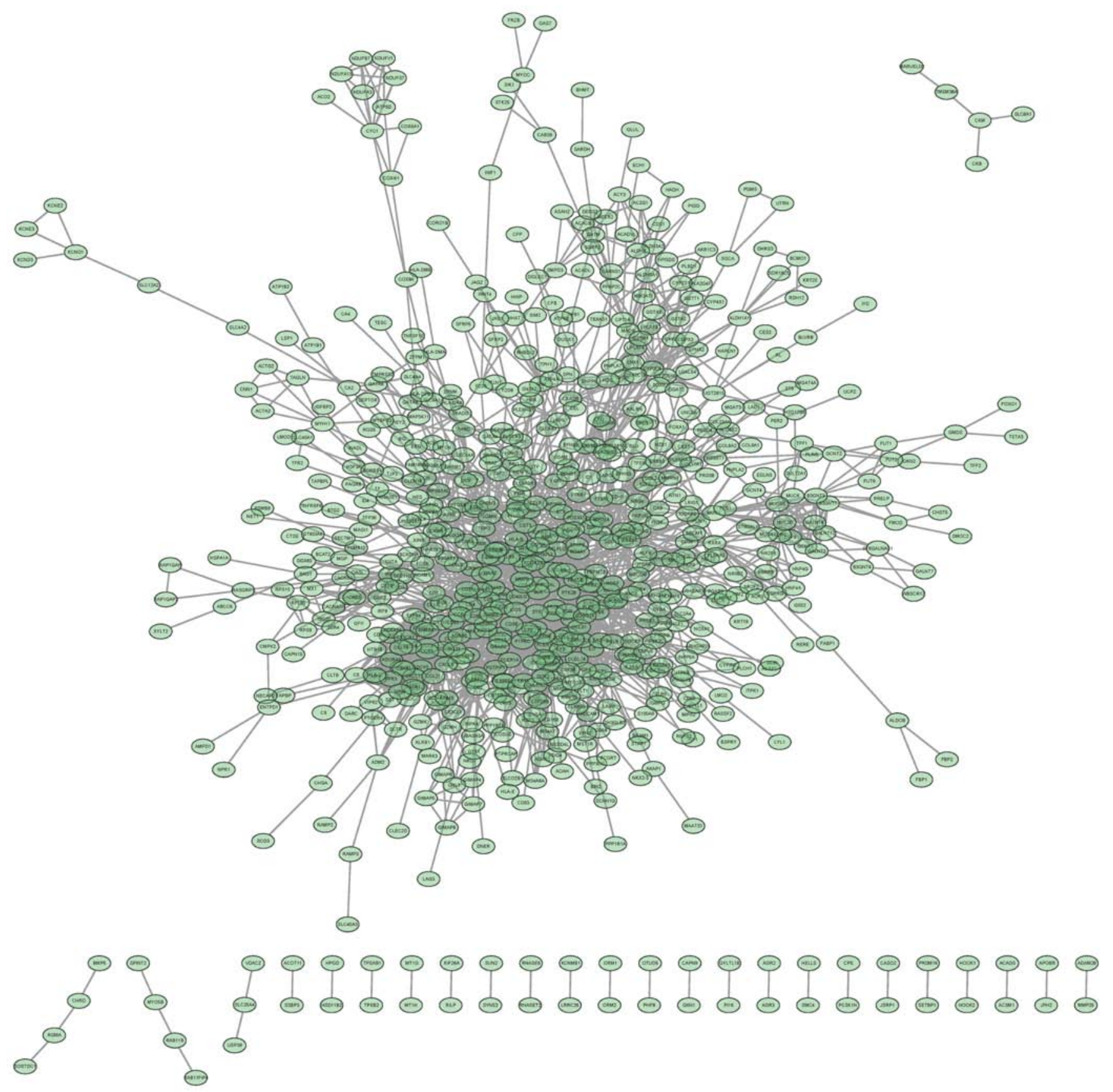

Figure 3. Protein-protein interaction network of the downregulated genes.

included ECM-receptor interaction and focal adhesion, both of which involved proteins encoded by $C O L$ and ITG genes. ECM-receptor interaction and focal adhesion are associated with cancer metastasis and aggression $(35,36)$, and can represent some molecular differences in gastric cancer (37). These observations may indicate that the $C O L$ and $I T G$ genes were associated with gastric cancer. In module A of the PPI network for proteins encoded by the upregulated genes, proteins encoded by $C O L$ genes were able to interact with those of $I T G$ genes, suggesting that $C O L$ genes are involved in gastric cancer through the regulation of ITG genes.

There is a high mutation probability of phosphoinositide-3-kinase, catalytic, $\alpha(P I K 3 C A)$ in human cancers and it is a potential therapy target for various tumors (38). The mutations of PIK3CA can lead to the attenuation of apoptosis and assist tumor invasion (39). The phosphatidylinositol 3-kinase (PI3K) pathway is of great alteration frequency in gastric tumors and can be used as a therapeutic target in gastric cancer (40). In module B of the PPI network for the downregulated genes, the enriched KEGG pathways for DEGs included the T-cell receptor signaling pathway, which involved proteins encoded by $P I K 3 C G$ and $P I K 3 R 5$. It has been reported that the $\mathrm{T}$-cell receptor signaling pathway plays a role in gastric cancer (41). The abovementioned findings showed that PIK3CG and PIK3R5 may be associated with gastric cancer. In module B of the PPI network for 

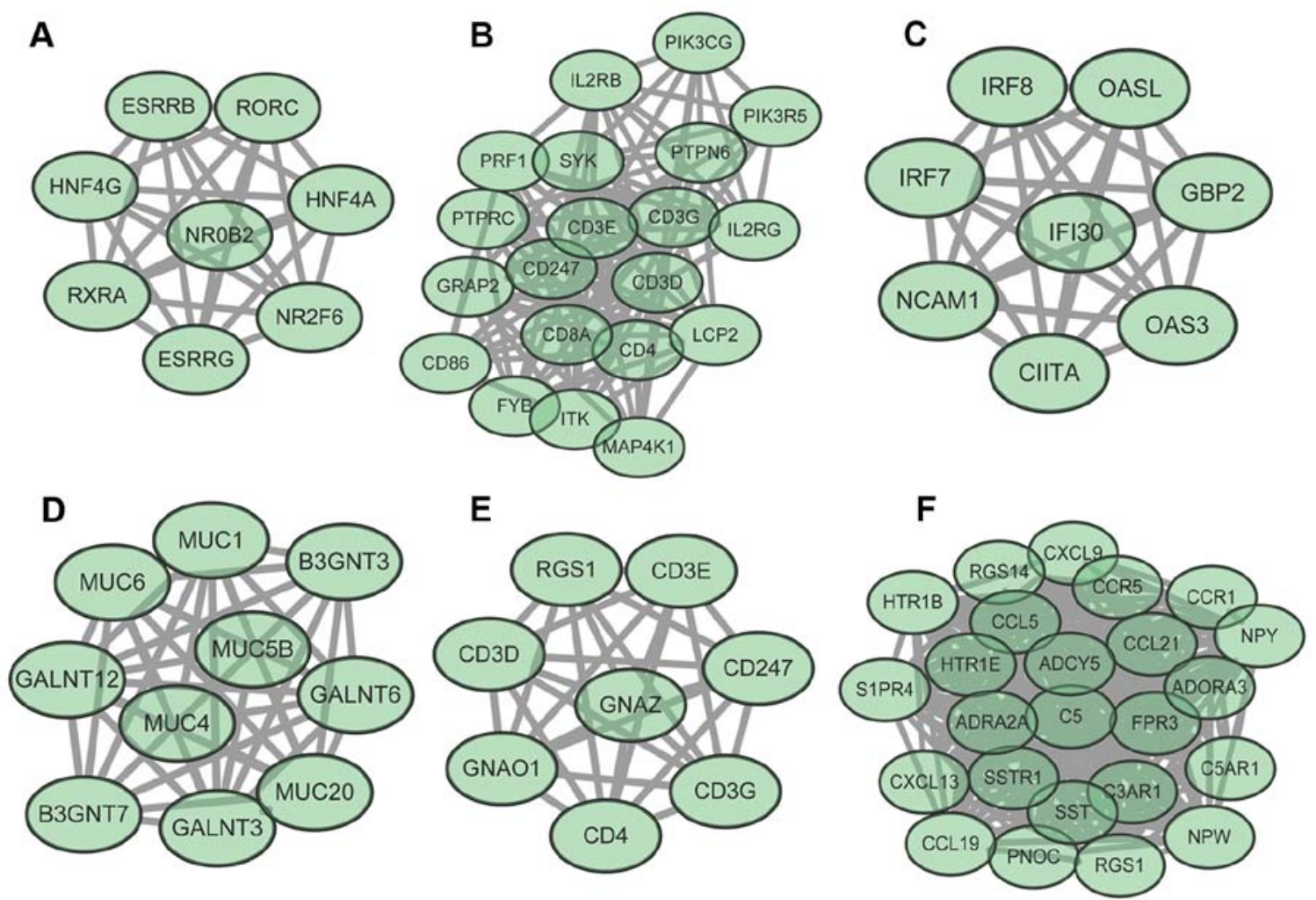

Figure 4. Module A-F obtained from the PPI network of the downregulated genes. PPI, protein-protein interaction.

Table IV. The top 10 enriched KEGG pathways for the DEGs co-expressed with lncRNA TCONS_00068220.

\begin{tabular}{|c|c|c|c|c|}
\hline ID & Name & Gene no. & Gene symbol & P-value \\
\hline KEGG:4514 & Cell adhesion molecules & 15 & $C D 2, C D 28 \ldots$ & $3.10 \mathrm{E}-06$ \\
\hline KEGG:4062 & Chemokine signaling pathway & 15 & $C C L 5, C C R 1 \ldots$ & 0.00020233 \\
\hline KEGG:5219 & Bladder cancer & 5 & $M M P 9, M Y C \ldots$ & 0.005609503 \\
\hline KEGG:4650 & Natural killer cell-mediated cytotoxicity & 8 & $B I D, I C A M 2 \ldots$ & 0.033796553 \\
\hline KEGG:4672 & Intestinal immune network for IgA production & 8 & $C D 28, I C O S L G \ldots$ & 4.17E-05 \\
\hline KEGG:5150 & Staphylococcus aureus infection & 8 & $C 1 Q B, C 1 Q C \ldots$ & 0.000114261 \\
\hline KEGG:5140 & Leishmaniasis & 9 & $I T G B 1, M A P K 12 \ldots$ & 0.000142588 \\
\hline KEGG:4940 & Type I diabetes mellitus & 7 & $C D 28, P R F 1 \ldots$ & 0.000149447 \\
\hline KEGG:5330 & Allograft rejection & 6 & $C D 28, H L A-D M B \ldots$ & 0.000460506 \\
\hline KEGG:5416 & Viral myocarditis & 8 & $B I D, C D 28 \ldots$ & 0.000623762 \\
\hline
\end{tabular}

KEGG, Kyoto Encyclopedia of Genes and Genomes; DEGs, differentially expressed genes.

proteins encoded by the downregulated genes, PIK3CG also had an interaction relationship with PIK3R5, indicating that $P I K 3 C G$ may be involved in gastric cancer by mediating PIK3R5.

The interleukin-8 (IL8) promoter polymorphism plays a role in atrophic gastritis and gastric cancer (42). Serum IL6 is correlated with the progression of gastric cancer and may be used as a biomarker for monitoring the treatment and response of patients with gastric cancer (43). Recombinant human $I L 7$ can retard tumor growth and induce complete regression (44). By activating p53 and inhibiting cell proliferation, IncRNA TCONS-00090092-MEG3 may act as a putative tumor-suppressor gene (45-47). In the present study, IL7 was co-expressed with TCONS-00068220. Proteins encoded with co-expressed DEGs of TCONS_00068220 were enriched in cancer-associated pathways. Thus, the expression levels of IL7 and TCONS-00068220 may be associated with gastric cancer, and $I L 7$ may function in gastric cancer by regulating TCONS-00068220.

In conclusion, we have conducted a comprehensive bioinformatics analysis of genes and lncRNAs that may be associated with gastric cancer. A total of 86 lncRNA transcriptions were obtained, as well as 1,088 upregulated and 1,537 downregulated transcriptions were screened. COL and ITG genes, 
PIK3CG, PIK3R5, IL7 and IncRNA TCONS-00068220 may be correlated with gastric cancer. However, investigations are to be conducted to determine the functional mechanisms of these genes in gastric cancer.

\section{References}

1. Kumar V, Abbas AK, Fausto N and Aster JC: Robbins and Cotran Pathologic Basis of Disease, Professional Edition: Expert Consult-Online. 8th edition, Elsevier Health Sciences, Philadelphia, PA, 2009.

2. Ruddon RW: Cancer Biology. 4th edition, Oxford University Press, New York, NY, 2007.

3. Parkin DM, Bray F, Ferlay J and Pisani P: Global cancer statistics, 2002. CA Cancer J Clin 55: 74-108, 2005.

4. Organization WHO: Are the number of cancer cases increasing or decreasing in the world? World Health Organization, Geneva, 2008.

5. Cancer IAfRo: World Cancer Report 2014. World Health Organization, Geneva, 2014.

6. Lozano R, Naghavi M, Foreman K, Lim S, Shibuya K, Aboyans V, Abraham J, Adair T, Aggarwal R, Ahn SY, et al: Global and regional mortality from 235 causes of death for 20 age groups in 1990 and 2010: a systematic analysis for the Global Burden of Disease Study 2010. Lancet 380: 2095-2128, 2012.

7. Organization WHO: Global battle against cancer won't be won with treatment alone effective prevention measures urgently needed to prevent cancer crisis. International Agency for Research on Cancer, Geneva, 2014.

8. Du JJ, Dou KF, Peng SY, Wang WZ, Wang ZH, Xiao HS, Guan WX, Liu YB and Gao ZQ: Down-regulated full-length novel gene GDDR and its effect on gastric cancer. Zhonghua Y Xue Za Zhi 83: 1166-1168, 2003 (In Chinese).

9. Moss SF, Lee J-W, Sabo E, Rubin AK, Rommel J, Westley BR, May FE, Gao J, Meitner PA, Tavares R, et al: Decreased expression of gastrokine 1 and the trefoil factor interacting protein TFIZ1/GKN2 in gastric cancer: Influence of tumor histology and relationship to prognosis. Clin Cancer Res 14 4161-4167, 2008

10. Fidan E, Mentese A, Ozdemir F, Deger O, Kavgaci H, Caner Karahan S and Aydin F: Diagnostic and prognostic significance of CA IX and suPAR in gastric cancer. Med Oncol 30: 540, 2013.

11. Meng LX, Li Q, Xue YJ, Guo RD, Zhang YQ and Song XY: Identification of gastric cancer-related genes by multiple high throughput analysis and data mining. Zhonghua Wei Chang Wai Ke Za Zhi 10: 169-172, 2007 (In Chinese).

12. Cao WJ, Wu HL, He BS, Zhang YS and Zhang ZY: Analysis of long non-coding RNA expression profiles in gastric cancer. World J Gastroenterol 19: 3658-3664, 2013.

13. Ponting CP, Oliver PL and Reik W: Evolution and functions of long noncoding RNAs. Cell 136: 629-641, 2009.

14. Sun M, Xia R, Jin F, Xu T, Liu Z, De W and Liu X: Downregulated long noncoding RNA MEG3 is associated with poor prognosis and promotes cell proliferation in gastric cancer. Tumour Biol 35: 1065-1073, 2014

15. Xu ZY, Yu QM, Du YA, Yang LT, Dong RZ, Huang L, Yu PF and Cheng XD: Knockdown of long non-coding RNA HOTAIR suppresses tumor invasion and reverses epithelial-mesenchymal transition in gastric cancer. Int J Biol Sci 9: 587-597, 2013.

16. Dai M, Thompson RC, Maher C, Contreras-Galindo R, Kaplan MH, Markovitz DM, Omenn G and Meng F: NGSQC: Cross-platform quality analysis pipeline for deep sequencing data. BMC Genomics 11 (Suppl 4): S7, 2010.

17. Kim D, Pertea G, Trapnell C, Pimentel H, Kelley R and Salzberg SL: TopHat2: Accurate alignment of transcriptomes in the presence of insertions, deletions and gene fusions. Genome Biol 14: R36, 2013.

18. Trapnell C, Roberts A, Goff L, Pertea G, Kim D, Kelley DR Pimentel H, Salzberg SL, Rinn JL and Pachter L: Differential gene and transcript expression analysis of RNA-seq experiments with TopHat and Cufflinks. Nat Protoc 7: 562-578, 2012.

19. Li B, Duan H, Li J, Deng XW, Yin W and Xia X: Global identification of miRNAs and targets in Populus euphratica under salt stress. Plant Mol Biol 81: 525-539, 2013.

20. Lin MF, Jungreis I and Kellis M: PhyloCSF: A comparative genomics method to distinguish protein coding and non-coding regions. Bioinformatics 27: i275-i282, 2011.
21. Bateman A, Coin L, Durbin R, Finn RD, Hollich V, GriffithsJones S, Khanna A, Marshall M, Moxon S, Sonnhammer EL, et al: The Pfam protein families database. Nucleic Acids Res 32: D138-D141, 2004.

22. Anders S, Reyes A and Huber W: Detecting differential usage of exons from RNA-seq data. Genome Res 22: 2008-2017, 2012.

23. Hulsegge I, Kommadath A and Smits MA: Globaltest and GOEAST: Two different approaches for Gene Ontology analysis. BMC Proc 3 (Suppl 4): S10, 2009.

24. Kanehisa M and Goto S: KEGG: Kyoto encyclopedia of genes and genomes. Nucleic Acids Res 28: 27-30, 2000.

25. Franceschini A, Szklarczyk D, Frankild S, Kuhn M, Simonovic M, Roth A, Lin J, Minguez P, Bork P, von Mering C, et al: STRING v9.1: Protein-protein interaction networks, with increased coverage and integration. Nucleic Acids Res 41 (D1): D808-D815, 2013.

26. Saito R, Smoot ME, Ono K, Ruscheinski J, Wang PL, Lotia S, Pico AR, Bader GD and Ideker T: A travel guide to Cytoscape plugins. Nat Methods 9: 1069-1076, 2012.

27. Adamcsek B, Palla G, Farkas IJ, Derényi I and Vicsek T: CFinder: Locating cliques and overlapping modules in biological networks. Bioinformatics 22: 1021-1023, 2006.

28. Schink M, Tröger W, Dabidian A, Goyert A, Scheuerecker H, Meyer J, Fischer IU and Glaser F: Mistletoe extract reduces the surgical suppression of natural killer cell activity in cancer patients. a randomized phase III trial. Forsch Komplementmed 14: 9-17, 2007.

29. Matsui H, Kubochi K, Okazaki I, Yoshino K, Ishibiki K and Kitajima M: Collagen biosynthesis in gastric cancer: Immunohistochemical analysis of prolyl 4-hydroxylase. J Surg Oncol 70: 239-246, 1999.

30. Wang ZN and Xu HM: Relationship between collagen IV expression and biological behavior of gastric cancer. World J Gastroenterol 6: 438-439, 2000.

31. David L, Nesland JM, Holm R and Sobrinho-Simões M: Expression of laminin, collagen IV, fibronectin, and type IV collagenase in gastric carcinoma. An immunohistochemical study of 87 patients. Cancer 73: 518-527, 1994.

32. Guszczyn T and Sobolewski K: Deregulation of collagen metabolism in human stomach cancer. Pathobiology 71: 308-313, 2004.

33. Kawamura T, Endo Y, Yonemura Y, Nojima N, Fujita H, Fujimura T, Obata T, Yamaguchi T and Sasaki T: Significance of integrin $\alpha 2 / \beta 1$ in peritoneal dissemination of a human gastric cancer xenograft model. Int J Oncol 18: 809-815, 2001.

34. Ishii Y, Ochiai A, Yamada T, Akimoto S, Yanagihara K, Kitajima $M$ and Hirohashi S: Integrin $\alpha 6 \beta 4$ as a suppressor and a predictive marker for peritoneal dissemination in human gastric cancer. Gastroenterology 118: 497-506, 2000.

35. Chiang AC and Massagué J: Molecular basis of metastasis. N Engl J Med 359: 2814-2823, 2008.

36. Joshi B, Strugnell SS, Goetz JG, Kojic LD, Cox ME, Griffith OL, Chan SK, Jones SJ, Leung SP, Masoudi H, et al: Phosphorylated caveolin-1 regulates Rho/ROCK-dependent focal adhesion dynamics and tumor cell migration and invasion. Cancer Res 68: 8210-8220, 2008.

37. Yang S, Shin J, Park KH, Jeung H-C, Rha SY, Noh SH, Yang WI and Chung HC: Molecular basis of the differences between normal and tumor tissues of gastric cancer. Biochim Biophys Acta 1772: 1033-1040, 2007.

38. Barbi S, Cataldo I, De Manzoni G, Bersani S, Lamba S, Mattuzzi S, Bardelli A and Scarpa A: The analysis of PIK3CA mutations in gastric carcinoma and metanalysis of literature suggest that exon-selectivity is a signature of cancer type. J Exp Clin Cancer Res: Apr 16, 2010. doi: 10.1186/1756-996629-32.

39. Samuels Y, Diaz LA Jr, Schmidt-Kittler O, Cummins JM, Delong L, Cheong I, Rago C, Huso DL, Lengauer C, Kinzler KW, et al: Mutant PIK3CA promotes cell growth and invasion of human cancer cells. Cancer Cell 7: 561-573, 2005.

40. Tran TN, Brettingham-Moore K, Duong CP, Mitchell C, Clemons NJ and Phillips WA: Molecular changes in the phosphatidylinositide 3-kinase (PI3K) pathway are common in gastric cancer. J Surg Oncol 108: 113-120, 2013.

41. Cho HJ, Baek KE, Park SM, Kim IK, Choi YL, Cho HJ, Nam IK, Hwang EM, Park JY, Han JY, et al: RhoGDI2 expression is associated with tumor growth and malignant progression of gastric cancer. Clin Cancer Res 15: 2612-2619, 2009. 
42. Taguchi A, Ohmiya N, Shirai K, Mabuchi N, Itoh A, Hirooka Y, Niwa $Y$ and Goto H: Interleukin-8 promoter polymorphism increases the risk of atrophic gastritis and gastric cancer in Japan. Cancer Epidemiol Biomarkers Prev 14: 2487-2493, 2005.

43. Wu CW, Wang SR, Chao MF, Wu TC, Lui WY, P'eng FK and Chi CW: Serum interleukin-6 levels reflect disease status of gastric cancer. Am J Gastroenterol 91: 1417-1422, 1996.

44. Vicari A, de Moraes MC, Gombert JM, Dy M, Penit C Papiernik $M$ and Herbelin A: Interleukin 7 induces preferential expansion of $\mathrm{V}$ beta $8.2^{+} \mathrm{CD} 48^{-}$and $\mathrm{V}$ beta $8.2^{+} \mathrm{CD} 4^{+} 8^{-}$murine thymocytes positively selected by class I molecules. J Exp Med 180: 653-661, 1994.
45. Zhang X, Rice K, Wang Y, Chen W, Zhong Y, Nakayama Y, Zhou Y and Klibanski A: Maternally expressed gene 3 (MEG3) noncoding ribonucleic acid: Isoform structure, expression, and functions. Endocrinology 151: 939-947, 2010.

46. Balik V, Srovnal J, Sulla I, Kalita O, Foltanova T, Vaverka M, Hrabalek L and Hajduch M: MEG3: A novel long noncoding potentially tumour-suppressing RNA in meningiomas. J Neurooncol 112: 1-8, 2013.

47. Qin R, Chen Z, Ding Y, Hao J, Hu J and Guo F: Long non-coding RNA MEG3 inhibits the proliferation of cervical carcinoma cells through the induction of cell cycle arrest and apoptosis. Neoplasma 60: 486-492, 2013. 